\title{
Avaliação da ocorrência de transtornos mentais comuns em usuários do Programa de Saúde da Família
}

\author{
Assessment of common mental disorders in the \\ Programa de Saúde da Família's users
}

Marina Bandeira', Lucas Cordeiro Freitas², João Gualberto Teixeira de Carvalho Filho ${ }^{3}$

\section{RESUMO}

Objetivo: Avaliar as características dos transtornos mentais comuns em usuários do Programa de Saúde da Família (PSF), visando a identificar a necessidade de atendimento em saúde mental. Método: Foram entrevistadas 400 pessoas indicadas por duas equipes do PSF, residentes de um bairro de baixo nível socioeconômico de uma cidade do interior de Minas Gerais. Entrevistadores previamente treinados aplicaram o Questionário de Saúde Mental (QSG), que avalia as características de transtornos mentais comuns não-psicóticos. Resultados: Obteve-se um índice global elevado (37,8\%) de pessoas com perfil sintomático indicativo de transtornos mentais comuns, destacando-se os sintomas referentes às Subescalas de Distúrbios do Sono (41\%) e Desejo de Morte (38,25\%) e menores índices nas Subescalas de Desconfiança do Próprio Desempenho (27,75\%), Distúrbios Psicossomáticos $(25,5 \%)$ e Estresse Psíquico (22\%). Houve taxa significativamente mais elevada no escore global para os homens, com 45,1\% apresentando perfil sintomático, comparativamente a $32,6 \%$ das mulheres. Porém, as mulheres apresentaram porcentagens mais elevadas de

\section{Palavras-chaves}

PSF, transtornos mentais comuns, saúde mental. perfil sintomático na subescala que se refere ao desejo de morte, $44,1 \%$ contra 29,9\% dos homens. Conclusão: Os resultados descrevem problemas importantes no estado de saúde mental, diferenciados por sexo, em usuários do PSF.

\section{ABSTRACT}

Objective: This study aimed at evaluating the characteristics of mental disturbances in a low socio-economic level sample of persons attended by a community family health program called Programa de Saúde da Família (PSF), in order to identify their need for care. Method: Trained research assistants interviewed 400 residents, indicated by the PSF team. Subjects were of a low economic level catchment area of a town in the interior of the state of Minas Gerais, in Brazil. Interviewers applied the Questionnaire of General Health (QSG), which evaluates the characteristics of mental health non-psychotic problems. Results: A high percentage of persons (37.8\%) had symptomatic global scores in the scale, which indicates the presence of problems in mental health, especially in Sub-scales Regarding Sleep Disturbances (41\%) and Desire of Death (38.25\%), and less in the Sub-scales of Distrust in Performance (27.75\%), Psychosomatic Disturbances

1 Professora adjunta da Universidade Federal de São João del-Rei (UFSJ), pesquisadora-bolsista do (NPq, Doutora Ph.D. pela Université de Montreal, com pós-doutorado pela McGill University e Université de Montreal. Carlos (UFSC) e bolsista Capes.

3 Professor adjunto do Departamento de Psicologia da UFSJ. 


\section{Key-words}

Mental health problems, family health service (FHS).
(25.5\%) and Psychological Stress (22\%). A significantly higher percentage of men had symptomatic global scores (45.1\%) compared to women (32.6\%), but a greater percentage of women (44.1\%) had symptomatic scores in the sub-scale regarding desire of death compared to men (29.9\%). Conclusion: The results obtained described important problems in mental health, differentiated by sex, and pointed to the need for mental health care of those persons.

\section{INTRODUÇÃO}

O Programa Saúde da Família (PSF) visa a desenvolver a prevenção e a promoção da saúde na comunidade, mediante uma ação assertiva com a população. Esse modelo de atuação prioriza a atenção primária à saúde, como alternativa a uma estratégia centrada exclusivamente na doença (Ministério da Saúde, 2006). No período de 1994 a 2005, o PSF foi implantado e disseminado por diversas regiões do país, chegando, em 2005, a uma cobertura que atendia 78,6 milhões de pessoas. O programa é desenvolvido por uma equipe básica composta por um médico de família, um enfermeiro, um auxiliar de enfermagem e seis agentes comunitários de saúde, podendo ser ampliada com a participação de um dentista, um auxiliar de consultório dentário e um técnico em higiene dental. A atuação das equipes do PSF ocorre nas unidades básicas de saúde e nas residências da população atendida, configurando-se como porta de entrada para o Sistema Único de Saúde (Ministério da Saúde, 2006).

Pesquisas têm sido realizadas, em âmbito nacional, e estudos multicêntricos, visando a avaliar diversos aspectos da implantação e do funcionamento do PSF, as percepções dos profissionais e usuários, assim como o impacto do programa na saúde da população. Quanto ao impacto na saúde, por exemplo, no que se refere à mortalidade infantil, um estudo multicêntrico amplo do Ministério da Saúde observou redução de 42\% de óbitos infantis no período de 1990 a 2002, em que o PSF já tinha sido implantado. No período seguinte, entre 2002 e 2004, queda de 14,69\% da mortalidade infantil foi observada nas regiões assistidas pelas equipes do PSF, principalmente em relação aos óbitos associados à diarréia e a infecções respiratórias (Brasil, 2005). No que se refere aos problemas de saúde relacionados ao diabetes e à hipertensão, um estudo multicêntrico (Aquino, 2004) observou associação positiva significativa entre a porcentagem de cobertura do PSF nos municípios brasileiros e a redução da taxa de internações por complicações dessas doenças, em indivíduos maiores de 20 anos, no período de 1998 a 2001, tendo-se controlado variáveis interferentes.

Estudos isolados realizados em municípios brasileiros também constataram efeito positivo do PSF em indicadores de saúde da população. Por exemplo, Mano e Pierin (2005), estudando dois grupos de 113 hipertensos, assistidos ou não pelo PSF, observaram que o grupo atendido pelo PSF apresentou uma diferença significativa na pressão sangüínea, em um período de 15 meses, o que não ocorreu com o grupo de comparação. Apesar de ter inicialmente apresentado valores superiores de pressão, o grupo assistido pelo PSF, no final do estudo, apresentou valores inferiores ao do grupo assistido pelo serviço tradicional de saúde. Em um outro estudo, constatou-se redução significativa de 44\% dos internamentos por acidente vascular cerebral (AVC), no município de Sobral, Ceará, entre os anos de 1997 e 2002, concomitantemente a um aumento do número de hipertensos acompanhados pelo PSF (Cruz et al., 2004). Em pesquisa a respeito da gravidez na adolescência, analisando o período de 1999 a 2001 e comparando populações atendidas ou não pelo PSF, em uma mesma cidade, observou-se proporção significativamente menor de casos na população atendida pelo PSF (Otsuka et al., 2005). O estudo de Guimarães (2004), realizado em Olinda, sobre o impacto do PSF no perfil epidemiológico infantil, em períodos de tempo analisados entre 1993 e 2000, detectou também redução de 17\% na prevalência de nascimentos com baixo peso, aumento na cobertura de vacinações e diminuição da mortalidade infantil. Em resumo, esses estudos observaram um impacto positivo da atuação do PSF em indicadores de saúde da população.

Entretanto, pesquisas avaliando problemas referentes à saúde mental da população atendida pelo PSF são menos freqüentes. A partir de uma busca no SciELO e Lilacs, poucas pesquisas publicadas em periódicos científicos foram identificadas, as quais investigaram o atendimento em saúde mental ou a ocorrência de transtornos mentais entre os usuários do PSF. Transtornos mentais ou sintomas psicológicos são freqüentes na população geral e entre pacientes de clínica médica, mas segundo a Organização Mundial da Saúde, são pouco identificados, referidos ou tratados e tendem a ser subestimados por profissionais de saúde, principalmente quando sintomas físicos estão presentes (WHO, 1994). No entanto, as características da acessibilidade e cobertura do PSF na população poderiam favorecer a detecção desses transtornos, assim como o encaminhamento dos pacientes para tratamento.

Alguns poucos estudos foram encontrados avaliando a ocorrência de transtornos mentais comuns (TMC) na população-alvo atendida pelo PSF. A denominação TMC se refere aos estados de saúde envolvendo sintomas psiquiátricos 
não-psicóticos, tais como sintomas depressivos, de ansiedade e psicossomáticos proeminentes, que trazem incapacidade funcional ou ruptura do funcionamento normal das pessoas, embora não preencham os critérios formais para diagnósticos de depressão e/ou ansiedade segundo as classificações do DSM-IV e da CID-10 (Maragno et al., 2006).

Na pesquisa realizada por Maragno et al. (2006), investigou-se a ocorrência de transtornos mentais comuns, utilizando a escala de rastreamento SRQ-20, elaborada pela Organização Mundial da Saúde $($ WHO, 1994) e validada para o Brasil por Mari e Williams (1986). Os resultados mostraram prevalência de $24,95 \%$ de pessoas com indicativos de transtornos mentais comuns, em dois distritos do município de São Paulo, avaliando populações que se situavam dentro e fora da área de cobertura do PSF. Não se verificaram diferenças significativas na prevalência de TMC entre as áreas dentro e fora dessa cobertura de serviço. Houve maior prevalência de TMC em pessoas do sexo feminino, em idosos com mais de 65 anos e em indivíduos com menor escolaridade e baixo nível socioeconômico. Em outro estudo, Cruz et al. (2005) detectaram prevalência ainda mais elevada de TMC, de $37,1 \%$, em uma amostra particular de 70 puérperas atendidas por duas unidades do PSF da Secretaria Municipal de Saúde da cidade de São Paulo, por meio da escala SRQ-20, paralelamente a uma taxa igualmente elevada de depressão puerperal, por meio da escala EPDS (Santos et al., 2000).

Em um estudo avaliando particularmente a sintomatologia depressiva, em 2.201 usuários de serviços de saúde primários de Porto Alegre, constatou-se um valor elevado da intensidade média de sintomatologia depressiva, que se situou acima do ponto de corte para homens e mulheres, em uma escala validada (CES-D). As mulheres apresentaram, neste estudo, intensidade mais elevada de sintomatologia depressiva. Verificou-se, ainda, relação inversa entre sintomas depressivos e indicadores do funcionamento psicológico e da qualidade de vida. Os usuários com mais sintomas depressivos utilizaram mais freqüentemente os serviços de saúde e permaneceram mais tempo internados do que os menos deprimidos (Fleck et al., 2002).

Tendo em vista a carência de investigações que avaliem o estado de saúde mental da população atendida pelo PSF, procurou-se realizar este estudo em uma região ainda não estudada, visando a ampliar o conhecimento dessa temática. O objetivo dessa pesquisa foi avaliar e descrever as características dos transtornos mentais comuns apresentados por indivíduos indicados por equipes do PSF.

\section{MÉTODO}

\section{Participantes}

Participaram deste estudo 400 indivíduos atendidos por duas equipes do programa PSF de uma cidade do interior de Minas Gerais. Os sujeitos habitavam um bairro da periferia da cidade, caracterizado por baixo nível socioeconômico. Os sujeitos foram apontados por essas equipes como indivíduos com indicativo de dificuldades psicológicas, tratando-se, portanto, de uma amostra pré-selecionada. A idade média dos sujeitos foi de 42,86 anos ( $d p=14,70)$, sendo 164 do sexo masculino e 236 do sexo feminino.

\section{Instrumento de medida}

Utilizou-se a Escala de Saúde Geral de Goldberg (QSG), originalmente elaborada por Goldberg (1972) e validada para o Brasil por Pasquali et al. (1996). Essa escala visa a avaliar a saúde mental das pessoas, por meio de itens que indicam a presença ou ausência de sintomas ou distúrbios clínicos não-psicóticos ou transtornos mentais comuns. O QSG permite identificar pessoas com perfil sintomático (com escores acima do percentil 90), casos limítrofes (entre 85 e 90) e pessoas sem perfil sintomático (abaixo de 85). Pessoas que apresentam perfil sintomático no GSG possuem índices desviantes de saúde mental e estado de disfunção que dificulta um funcionamento adequado. Esse estado incapacita as pessoas a terem vidas pessoal e social satisfatórias.

Trata-se de uma escala multifatorial, contendo cinco Subescalas: 1. Estresse Psíquico; 2. Desejo de Morte; 3. Desconfiança do Próprio Desempenho; 4. Distúrbios do Sono; 5. Distúrbios Psicossomáticos. Fornece ainda um escore global que indica a severidade de problemas de saúde mental. A escala possui 60 itens, com alternativas de respostas em escala do tipo Likert de quatro pontos, variando de "menos do que de costume" (ponto 1) a "muito mais que de costume" (ponto 4), quando o item é formulado como sintoma e o sentido inverso, quando o item expressa comportamento normal.

\section{Procedimento}

Os sujeitos foram entrevistados por 10 estagiários de psicologia previamente treinados. Esse treinamento constou de entrevistas simuladas e supervisionadas pelos pesquisadores. Após o treinamento, a coleta de dados foi feita por meio de entrevistas estruturadas nos domicílios dos sujeitos, tendo a equipe do PSF intermediado o acesso dos estagiários à população-alvo. As entrevistas visavam à aplicação da Escala de Saúde Geral, de Goldberg, e tinham duração aproximada de 45 minutos. Os dados foram analisados por meio do programa SPSS, versão 7.5. Essa pesquisa foi aprovada pelo Comitê de Ética da Instituição.

\section{RESULTADOS}

Os resultados obtidos estão apresentados nas tabelas 1 e 2 . A tabela 1 apresenta os dados referentes à amostra total dos sujeitos e, nela, pode-se observar que a amostra apresentou 
um índice global elevado (37,8\%) de pessoas com perfil sintomático do QSG, indicativo de transtornos na saúde mental. Quanto às Subescalas do QSG, os resultados mostram porcentagens mais elevadas de pessoas com perfil sintomático em duas subescalas: $41 \%$ na Subescala de Distúrbios do Sono e 38,25\% na Escala Referente ao Desejo de Morte. Nas demais subescalas, as porcentagens de pessoas com perfil sintomático, em ordem decrescente, foram menores: 27,75\% na Subescala de Desconfiança do Próprio Desempenho, 25,5\% na Subescala Referente a Distúrbios Psicossomáticos e 22\% na Subescala de Estresse Psíquico.

Os resultados diferenciados por gênero estão apresentados na tabela 2. Observa-se, nessa tabela, no que se refere à Escala Global do QSG, uma porcentagem de 45,1\% de homens com perfil sintomático, comparativamente a 32,6\% das mulheres.

Tabela 1. Número e porcentagem de sujeitos da amostra total, classificados em cada categoria do perfil avaliado, nos fatores e no escore de saúde geral da Escala QSG

\begin{tabular}{|c|c|c|c|}
\hline Fator & $\begin{array}{c}\text { Perfil } \\
\text { sintomático }\end{array}$ & $\begin{array}{c}\text { № de } \\
\text { sujeitos (n) }\end{array}$ & $\begin{array}{c}\text { Porcentagem } \\
(\%)\end{array}$ \\
\hline \multirow{4}{*}{ Estresse psíquico } & Sem sintomas & 247 & 61,8 \\
\hline & Casos limítrofes & 65 & 16,3 \\
\hline & Perfil sintomático & 88 & 22,0 \\
\hline & TOTAL & 400 & 100 \\
\hline \multirow{4}{*}{ Desejo de morte } & Sem sintomas & 201 & 50,3 \\
\hline & Casos limítrofes & 46 & 11,5 \\
\hline & Perfil sintomático & 153 & 38,3 \\
\hline & TOTAL & 400 & 100 \\
\hline \multirow{4}{*}{$\begin{array}{l}\text { Desconfiança do } \\
\text { próprio desempenho }\end{array}$} & Sem sintomas & 247 & 61,8 \\
\hline & Casos limítrofes & 42 & 10,5 \\
\hline & Perfil sintomático & 111 & 27,8 \\
\hline & TOTAL & 400 & 100 \\
\hline \multirow{4}{*}{ Distúrbios do sono } & Sem sintomas & 177 & 44,3 \\
\hline & Casos limítrofes & 59 & 14,8 \\
\hline & Perfil sintomático & 164 & 41,0 \\
\hline & TOTAL & 400 & 100 \\
\hline \multirow{4}{*}{$\begin{array}{l}\text { Distúrbios } \\
\text { psicossomáticos }\end{array}$} & Sem sintomas & 267 & 66,8 \\
\hline & Casos limítrofes & 31 & 7,8 \\
\hline & Perfil sintomático & 102 & 25,5 \\
\hline & TOTAL & 400 & 100 \\
\hline \multirow{4}{*}{ Saúde geral } & Sem sintomas & 205 & 51,3 \\
\hline & Casos limítrofes & 44 & 11,0 \\
\hline & Perfil sintomático & 151 & 37,8 \\
\hline & TOTAL & 400 & 100 \\
\hline
\end{tabular}

Quanto às subescalas, os resultados diferiram em função do sexo, com os homens apresentando maiores porcentagens em grande parte delas. As porcentagens observadas foram as seguintes: 59,1\% dos homens apresentaram perfil sintomático na Subescala de Distúrbios do Sono, contra 28,4\% das mulheres, 39,6\% na Subescala Distúrbios Psicossomáticos, comparativamente a 15,7\% das mulheres, 34,1\% na Subescala de Estresse
Tabela 2. Número e porcentagem de homens e mulheres, para cada categoria do perfil avaliado nos fatores, e escore geral de saúde da Escala QSG

\begin{tabular}{|c|c|c|c|c|}
\hline Fator & Gênero & $\begin{array}{c}\text { Perfil } \\
\text { sintomático }\end{array}$ & $\begin{array}{c}\text { № de } \\
\text { sujeitos } \\
\text { (n) }\end{array}$ & $\begin{array}{c}\text { Porcentagem } \\
(\%)\end{array}$ \\
\hline \multirow{8}{*}{$\begin{array}{l}\text { Estresse } \\
\text { psíquico** }\end{array}$} & \multirow{3}{*}{ Homens } & Sem sintomas & 77 & 47,0 \\
\hline & & Casos limítrofes & 31 & 18,9 \\
\hline & & Perfil sintomático & 56 & 34,1 \\
\hline & \multirow{5}{*}{ Mulheres } & TOTAL & 164 & 100 \\
\hline & & Sem sintomas & 170 & 72,0 \\
\hline & & Casos limítrofes & 34 & 14,4 \\
\hline & & Perfil sintomático & 32 & 13,6 \\
\hline & & TOTAL & 236 & 100 \\
\hline \multirow{8}{*}{$\begin{array}{l}\text { Desejo } \\
\text { de morte** }\end{array}$} & \multirow{3}{*}{ Homens } & Sem sintomas & 79 & 48,2 \\
\hline & & Casos limítrofes & 36 & 22,0 \\
\hline & & Perfil sintomático & 49 & 29,9 \\
\hline & \multirow{5}{*}{ Mulheres } & TOTAL & 164 & 100 \\
\hline & & Sem sintomas & 122 & 51,7 \\
\hline & & Casos limítrofes & 10 & 4,2 \\
\hline & & Perfil sintomático & 104 & 44,1 \\
\hline & & TOTAL & 236 & 100 \\
\hline \multirow{8}{*}{$\begin{array}{l}\text { Desconfiança } \\
\text { do próprio } \\
\text { desempenho* }\end{array}$} & \multirow{3}{*}{ Homens } & Sem sintomas & 90 & 54,9 \\
\hline & & Casos limítrofes & 13 & 7,9 \\
\hline & & Perfil sintomático & 61 & 37,2 \\
\hline & \multirow{5}{*}{ Mulheres } & TOTAL & 164 & 100 \\
\hline & & Sem sintomas & 157 & 66,5 \\
\hline & & Casos limítrofes & 29 & 12,3 \\
\hline & & Perfil sintomático & 50 & 21,2 \\
\hline & & TOTAL & 236 & 100 \\
\hline \multirow{8}{*}{$\begin{array}{l}\text { Distúrbios } \\
\text { do sono** }\end{array}$} & \multirow{3}{*}{ Homens } & Sem sintomas & 55 & 33,5 \\
\hline & & Casos limítrofes & 12 & 7,3 \\
\hline & & Perfil sintomático & 97 & 59,1 \\
\hline & \multirow{5}{*}{ Mulheres } & TOTAL & 164 & 100 \\
\hline & & Sem sintomas & 122 & 51,7 \\
\hline & & Casos limítrofes & 47 & 19,9 \\
\hline & & Perfil sintomático & 67 & 28,4 \\
\hline & & TOTAL & 236 & 100 \\
\hline \multirow{8}{*}{$\begin{array}{l}\text { Distúrbios } \\
\text { psicossomáticos** }\end{array}$} & \multirow{3}{*}{ Homens } & Sem sintomas & 88 & 53,7 \\
\hline & & Casos limítrofes & 11 & 6,7 \\
\hline & & Perfil sintomático & 65 & 39,6 \\
\hline & \multirow{5}{*}{ Mulheres } & TOTAL & 164 & 100 \\
\hline & & Sem sintomas & 179 & 75,8 \\
\hline & & Casos limítrofes & 20 & 8,5 \\
\hline & & Perfil sintomático & 37 & 15,7 \\
\hline & & TOTAL & 236 & 100 \\
\hline \multirow{8}{*}{ Saúde geral * } & \multirow{3}{*}{ Homens } & Sem sintomas & 70 & 42,7 \\
\hline & & Casos limítrofes & 20 & 12,2 \\
\hline & & Perfil sintomático & 74 & 45,1 \\
\hline & \multirow{5}{*}{ Mulheres } & TOTAL & 164 & 100 \\
\hline & & Sem sintomas & 135 & 57,2 \\
\hline & & Casos limítrofes & 24 & 10,2 \\
\hline & & Perfil sintomático & 77 & 32,6 \\
\hline & & TOTAL & 236 & 100 \\
\hline
\end{tabular}

${ }^{*} p<0,05 ;{ }^{* *} p<0,001$

Psíquico contra $13,6 \%$ das mulheres, $37,2 \%$ na Subescala Referente à Desconfiança do Próprio Desempenho contra 21,2\% das mulheres. Por outro lado, as mulheres apresentaram porcentagens mais elevadas de perfil sintomático na Subescala Referente ao Desejo de Morte (44,1\% contra 29,9\%).

Uma análise estatística, por meio do Qui-Quadrado, mostrou haver uma diferença significativa entre homens e mulheres com relação à distribuição das porcentagens 
de sujeitos nas categorias avaliadas pelo QSG. Os homens apresentaram porcentagens significativamente mais elevadas comparativamente às mulheres, na escala global e na maioria das subescalas.

\section{DISCUSSÃO}

Os resultados obtidos na presente pesquisa apontam para a ocorrência de problemas importantes no estado de saúde mental da amostra estudada. Constatou-se que, entre os indivíduos apontados pelas equipes do PSF, houve, de fato, uma porcentagem elevada de pessoas que apresentam indicadores de sintomas de transtornos mentais não-psicóticos, nas cinco dimensões avaliadas pela Escala QSG e no escore global. Verificaram-se maiores porcentagens de pessoas com transtornos do sono e desejo de morte e menor ocorrência de outros tipos de transtornos (distúrbios psicossomáticos, estresse psíquico e desconfiança do próprio desempenho). As características dos transtornos mentais, distribuídos por sexo, mostraram que os homens apresentaram maior porcentagem de transtornos, particularmente do sono, enquanto as mulheres se destacaram com transtornos referentes ao desejo de morte. Esses dados apontam para problemáticas distintas em função do sexo que requerem atendimentos e encaminhamentos diferenciados, assim como a necessidade de aprofundar o conhecimento sobre diferenças entre grupos da população-alvo. A elevada ocorrência de distúrbios do sono entre os homens da amostra é preocupante, pois essa sintomatologia tem sido associada a transtornos psiquiátricos (Loayza et al., 2001).

Os resultados da presente pesquisa ampliam os dados de outros estudos, descrevendo os tipos de transtornos mentais ocorridos. Entretanto, uma comparação desses resultados com os outros estudos não seria possível, uma vez que esse tipo de descrição não foi encontrado nas demais pesquisas, provavelmente em razão das limitações nos instrumentos de medida utilizados. A escala multifatorial QSG, utilizada no presente trabalho, contém diferentes subescalas, o que permite a descrição dos diferentes tipos de transtornos ocorridos.

Quanto à porcentagem de casos observados, no estudo de Maragno et al. (2006), identificou-se uma ocorrência de 24,95\% de transtornos mentais comuns nas populações estudadas e essa ocorrência foi maior para as mulheres (27,99\% contra 20,94\%). Essa taxa global mais baixa do que a obtida no presente trabalho explica-se pela amostragem aleatória do estudo de Maragno et al. (2006), contrariamente à da presente pesquisa. Quanto à diferenciação por sexo, esses dados estão em acordo com o presente estudo apenas no que se refere a uma das subescalas do QSG, que é a de Desejo de Morte, mas não para a escala global, que indicou maior taxa de TMC para os homens, no presente trabalho. Entretanto, a diferença entre as amostragens dos dois estudos possa, talvez, explicar essa diferença de resultados.

A taxa encontrada no presente trabalho se assemelha à obtida no estudo de Cruz et al. (2005), que identificou a ocorrência de 37,1\% de TMC, em uma amostra específica de puérperas de duas unidades do PSF. A concordância entre esses dois estudos pode ser explicada considerandose que mulheres no período puerperal constituem uma amostra vulnerável, assim como a amostra do presente trabalho poderia também ser considerada vulnerável, uma vez que foi indicada pelo PSF. O estudo de Fleck et al. (2002) apontou igualmente para a ocorrência de uma intensidade média elevada da sintomatologia depressiva em usuários de serviços primários de saúde, no Sul do país, tendo observado um escore significativamente mais elevado para as mulheres. Esses dados concordam com os resultados da presente pesquisa, uma vez que se observou também um resultado mais elevado para as mulheres, na Subescala Desejo de Morte.

Resultados desse tipo indicam a necessidade de focalizar o atendimento do PSF também para questões de saúde mental da população, em termos de detecção de sintomas e do atendimento inicial, sob forma de informações e orientações, assim como encaminhamento para outros serviços. Outros autores apontaram para tal necessidade. Por exemplo, Koga et al. (2006), investigando o atendimento em saúde mental do PSF em uma cidade do interior de São Paulo, compararam os dados de questionários respondidos por profissionais, pacientes e familiares, em uma amostra de 142 sujeitos, e constataram que embora o programa tenha se mostrado eficiente na tarefa de agendamento de consultas, apresentou dificuldades em prover informações e orientações necessárias a pacientes psiquiátricos e aos familiares cuidadores desses pacientes. No entanto, sabe-se que esses familiares sofrem de sobrecarga decorrente do papel de cuidador, que pode resultar em níveis elevados de estresse e transtornos mentais comuns, com sintomas de ansiedade e depressão (Bandeira e Barroso, 2005; Tessler e Gamache, 2000; Maurin e Boyd, 1990). No estudo de Koga et al. (2006), concluiu-se que os profissionais do PSF precisam receber melhor preparação para atender os pacientes com problemas de saúde mental e para orientar seus familiares cuidadores e que o programa PSF necessita se ampliar e se integrar aos demais programas de saúde, em particular o de saúde mental.

Os estudos de Nascimento e Braga (2004) e de Souza e Scatena (2005) apontaram para essas mesmas conclusões, a partir de dados coletados por meio de entrevistas semi-estruturadas e grupos focais com profissionais do PSF, respectivamente. Koga et al. (2006) destacaram a necessidade de a equipe do PSF fornecer informações sobre os medicamentos psiquiátricos, dar orientações e suporte aos familiares cuidadores, visando a diminuir sua sobrecarga e 
orientar os familiares sobre como lidar com os pacientes. Os autores destacam, ainda, a necessidade de intervir visando a aumentar a participação ativa e a responsabilidade dos pacientes com o próprio tratamento, a fim de evitar o abandono dos medicamentos e as conseqüentes reospitalizações repetidas.

A OMS tem recomendado uma ação educativa com os profissionais de saúde geral, visando a aumentar sua sensibilidade a sintomas psicológicos, indicativos de problemas de saúde mental, apresentados pela população ou por pacientes da clínica de medicina geral (WHO, 1994). No presente trabalho, a taxa de detecção desses problemas foi elevada na amostra indicada pelo PSF, mas seria provavelmente maior se uma ação educativa, tal como recomendada pela OMS, fosse implementada. Além disso, uma ação de acolhimento inicial da população para queixas de saúde mental, orientações e informações básicas, assim como triagem para encaminhamento aos serviços locais disponíveis, também constitui intervenção importante, que poderia alcançar maior cobertura pelas equipes do PSF.

$\mathrm{O}$ acolhimento inicial e a triagem foram incrementados em um programa de PSF, em uma cidade do interior de Minas Gerais, a partir da experiência de participação de estagiários de psicologia, supervisionados no âmbito do estágio do Internato Rural da UFMG, descritos no trabalho de Souza e Carvalho (2003). Segundo os autores, os estagiários incorporaram, ao trabalho do PSF, atendimentos psicológicos de acolhimento inicial e triagem, que permitiram estabelecer uma ordem de prioridade de encaminhamentos e atendimentos. Também se realizaram intervenções educativas, de sensibilização e incentivo à autonomia e à responsabilização do paciente com relação ao seu estado de saúde, em grupos programáticos de diabéticos, hipertensos, gestantes, terceira idade e jovens. Os resultados mostraram que a experiência serviu para promover maior agilidade do serviço, diminuição do tempo de demora para atendimento, priorização e organização da demanda (Souza e Carvalho, 2003).

Os resultados do presente trabalho se restringem a uma amostra limitada e previamente indicada. Pesquisas futuras deverão aprofundar o conhecimento sobre as características dos transtornos mentais apresentados por essa população-alvo de usuários do PSF ou de outros serviços de saúde, mediante uma amostragem aleatória e de maior amplitude.

\section{CONCLUSÃO}

A presente pesquisa apresentou resultados de uma região diferente dos estudos citados, ampliando assim o conhecimento a respeito dos usuários atendidos pelo PSF, no que se refere à saúde mental, em termos das característi- cas dos transtornos mentais observados. Pesquisas futuras, em regiões diversificadas do nosso país, poderão avaliar a amplitude dos problemas de saúde mental, aprofundar a descrição dos tipos de transtornos ocorridos e identificar a necessidade de atendimento dessa problemática.

\section{REFERÊNCIAS}

Aquino R. Avaliando os Efeitos do Programa de Saúde da Família sobre a Redução nas Internações por Complicações de Hipertensão e Diabetes em Municípios Brasileiros. Anais do VI Congresso Brasileiro de Epidemiologia, Recife, 2004.

Bandeira M, Barroso S. Sobrecarga das famílias de pacientes psiquiátricos. Jornal Brasileiro de Psiquiatria, 54 (1): 34-46, 2005.

Brasil, Ministério da Saúde. Mortalidade infantil cai mais de 14\% nas áreas cobertas pelo Programa Saúde da Família. Boletim em questão, n 330, julho (20/06/2005). Disponível em $<$ http://portal.saude.gov.br/portal/aplicacoes/noticias/noticias_detalhe.cfm?co_seq_ noticia $=16768>$, 2005. Acessado em 22/10/2006.

Cruz EBS, Simões GL, Faisal-Cury A. Rastreamento da depressão pós-parto em mulheres atendidas pelo Programa de Saúde da Família. Revista Brasileira de Ginecologia e Obstetrícia, 27 (4): 181-8, 2005.

Cruz NMG, Filho AAA, Barreto ICHC, Andrade LOM, Dias F, Torquato NFL, Amâncio CPA, Oliveira SML. Atuação do Programa Saúde da Família na Redução dos Internamentos por Acidente Vascular Cerebral em Sobral (CE). Anais do VI Congresso Brasileiro de Epidemiologia, Recife, 2004.

Fleck MPA, Lima AFBS, Louzada S, Schestasky G, Henriques A, Borges VR, Camey S. Associação entre sintomas depressivos e funcionamento social em cuidados primários à saúde. Rev Saúde Pública, 36 (4): 431-8, 2002.

Guimarães TMR. Avaliação do Impacto do PSF em Olinda. Anais do VI Congresso Brasileiro de Epidemiologia e 6a Reunião Científica da América Latina e Caribe da Associação Internacional de Epidemiologia (IEA), 2004, Recife. Revista Brasileira de Epidemiologia-ABRASCO. Caucaia-Ceará: Nordeste Digital Line S/A, 2004.

Goldberg DP. The Detection of Psychiatric IIIness by Questionnaire: a Technique for the Identification and Assessment of Non-psychotic Psychiatric IIIness. London: Oxford University Press; 1972.

Koga M, Furegato ARF, Santos JFL. Opinions of the staff and users about the quality of the mental health care delivered at a family health program. Rev Latino-Am Enfermagem 14 (2): 163-9, 2006.

Loayza MPH, Ponte TS, Carvalho CG, Pedrotti MR, Nunes PV, Souza CM, Zanette CB, Voltolini S, Chaves MLF. Association between mental health screening by self-report questionnaire and insomnia in medical students. Arq Neuropsiquiatr, 59 (2-A): 180-5, 2001.

Mano GMP, Pierin AMG. Avaliação de pacientes hipertensos acompanhados pelo Programa Saúde da Família em um Centro de Saúde. Acta Paul Enfermagem, 18(3): 269-75, 2005.

Maragno L, Goldbaum M, Gianini RJ, Novaes HMD, César CLC. Prevalência de transtornos mentais comuns em populações atendidas pelo Programa Saúde da Família (QUALIS) no Município de São Paulo. Cad Saúde Pública, 22 (8): 1639-48, 2006.

Mari JJ, Williams P. A validity study of a psychiatric screening questionnaire (SRQ-20) in primary care in the city of São Paulo. Br J Psychiatry, 148: 23-6, 1986.

Maurin JT, Boyd CB. Burden of mental illness on the family: a critical review. Archives of Psychiatric Nursing, 4(2): 99-107, 1990.

Ministério da Saúde. Departamento de Atenção Básica (2006). Atenção Básica e a Saúde da Família. Disponível em: <http://dtr2004.saude.gov.br/dab/atencaobasica.php>. Acessado em: 22/10/2006.

Nascimento AM, Braga VAB. Atenção em saúde mental: a prática do enfermeiro e do médico do Programa Saúde da Família de Caucaia. Cogiatare Enferm, 9 (1): 84-93, 2004.

Otsuka F, Narahara J, Ayabe L, Caccelli M, Salina V, Molinos V, Martins LC, Luiz OC. 0 programa de saúde da família e a gravidez na adolescência em São Bernardo do Campo. Arq Méd $A B C, 30(2): 90-3,2005$. 
Pasquali L, Gouveia VV, Andriola WB, Miranda FJ, Ramos ALM (1996). Questionário de Saúde Geral de Goldberg (QSG): adaptação brasileira. Psicologia: Teoria e Pesquisa, 10: 421-37, 1996.

Santos MFS, Martins FC, Pasquali L. Escala de auto-registro de depressão pós-parto. In: Gorenstein C, Andrade LHS, Zuardi AW (orgs.). Escalas de Avaliação Clínica em Psiquiatria e Psicofarmacologia. Lemos, p. 97-103, São Paulo, 2000.

Souza RA, Carvalho AM. Programa de saúde da família e qualidade de vida: um olhar da Psicologia. Estudos de Psicologia, 8 (3): 515-23, 2003
Souza RC, Scatena MCM. Produção de sentidos acerca da família que convive com o doente mental. Rev Latino-Am Enfermagem 13 (2): 173-79, 2005.

Tessler RC, Gamache GM. Family Experiences with Mental IIIness. Westport: Auburn House; 2000.

WHO. A user's guide to the Self-Reporting Questionnaire (SRQ-20). Division of Mental Health. World Organization, Geneva, 1994. 